\title{
Relación entre la Glucemia Maternal y Fetal y el Páncreas Endocrino Fetal en Alpacas
}

\author{
Relationship between Maternal and Foetal Glycemia and Foetal Endocrine \\ Pancreas in Alpacas
}

\author{
Johan Huaynates O. ${ }^{1}$, Juan Espinoza B. ${ }^{1}$, Bernardo López-Torres ${ }^{3}$, \\ Alejandro Rodríguez G. ${ }^{3,5}$, César Caro M. ${ }^{4}$, José Rodríguez G. ${ }^{2,6}$
}

\section{Resumen}

El presente trabajo tuvo por objetivo relacionar la glucemia maternal gestacional con la glucemia fetal y el desarrollo del páncreas endocrino fetal. Se utilizaron 18 alpacas gestantes y sus respectivos fetos. Lo animales y los fetos se distribuyeron en los tres tercios del periodo de gestación $(n=3,4$ y 11 para el primer, segundo y tercer tercio, respectivamente). La relación entre los niveles de glucosa maternal y los niveles de glucosa fetal, número de islotes de Langerhans (IL) por área $\left(\mathrm{N}^{\circ} \mathrm{IL} / \mathrm{mm}^{2}\right.$ fetal y diámetro de los IL fetal fue de r: 0.98, 0.99, 0.93, respectivamente. Los niveles de glucosa fetalmaternal, $\mathrm{N}^{\circ} \mathrm{IL} / \mathrm{mm}^{2}$ y diámetro de los IL fueron significativamente mayores en el último tercio de gestación $(\mathrm{p}<0.001)$. Los resultados indicarían que hay una influencia de la glucosa maternal en el desarrollo del páncreas endocrino fetal.

Palabras clave: glucemia materno-fetal, páncreas endocrino fetal

\section{Abstract}

The aim of this study was determine the relationship between maternal glycemia with foetal glycemia and development of foetal endocrine pancreas. Eighteen pregnant alpacas and their respective foetus were grouped in the three thirds of the gestation period ( $\mathrm{n}=$ 3, 4 and 11 for first, second and third third respectively). The relationship between levels of maternal glycemia and the levels of foetal glycemia, number of Langerhans islets (IL)

${ }^{1}$ Laboratorio de Farmacología y Toxicología, Facultad de Medicina Veterinaria Universidad Nacional Mayor de San Marcos, Lima, Perú

${ }^{2}$ Estación Experimental del Centro de Investigación IVITA-El Mantaro, Universidad Nacional Mayor de San Marcos, Huancayo, Perú

${ }^{3}$ SENASA Región Junín, Perú

${ }^{4}$ Laboratorio de Patología, SENASA, Lima, Perú

${ }^{5}$ E-mail: alexpolrodriguez@gmail.com

${ }^{6}$ E-mail: joserodriguezmv@gmail.com

Recibido: 29 de mayo de 2015

Aceptado para publicación: 6 de setiembre de 2015 
per area $\left(\mathrm{N}^{\circ} \mathrm{IL} / \mathrm{mm}^{2}\right)$ and diameter of IL were of r: 0.98, 0.99, 0.93, respectively. Levels of maternal-foetal glucose, $\mathrm{N}^{\circ} \mathrm{IL} / \mathrm{mm}^{2}$ and IL diameter were significantly higher $(\mathrm{p}<0.001)$ in the last third of gestation. The results would show that maternal glycemia affects the development of foetal endocrine pancreas.

Key words: maternal-foetal glycemia, foetal endocrine pancreas

\section{INTRODUCCIÓN}

Algunos estudios realizados en camélidos sobre el metabolismo de la glucosa reportan diferencias con otras especies. Es así que en contraste con los rumiantes, las llamas y las alpacas mantienen una alta concentración de glucosa en sangre (media: 7.0 $\mathrm{mmol} / \mathrm{l}$; rango: 4.6-8.9 $\mathrm{mmol} / \mathrm{l})$, semejante a un animal no rumiante (Lassen et al., 1986; Fowler y Zinkl, 1989; Kaneko, 1989). Las llamas y las alpacas también muestran respuestas hiperglicémicas extremas (concentraciones de glucosa en sangre de 11.1 a $16.6 \mathrm{mmol} / \mathrm{l}$ ) en respuesta a situaciones de estrés (Fowler y Zinkl, 1989; Cebra et al., 2001a,b). El elevado nivel de glucosa en sangre podría ser explicado por una lenta y moderada resistencia a la insulina que se observa en estas especies, característica similar a una condición de diabetes en el humano (Cebra et al., 2001a,b).

No se disponen de estudios relacionados al metabolismo energético en la etapa fetal de los camélidos sudamericanos (CSA); sin embargo, se puede considerar que la morfogénesis y la diferenciación del páncreas y sus tipos celulares son muy conservados entre los vertebrados durante el desarrollo fetal (Noden y Lahunta, 1990). El páncreas se desarrolla como estructura de yemas simples en el tubo del intestino primitivo a un órgano muy ramificado con diversos tipos de células especializadas (Kim y MacDonald, 2002). El páncreas maduro es un órgano bifuncional constituido principalmente por tejido exocrino organizado en acinos que segregan zimógenos en la luz intestinal, en tanto que la otra porción pancreática, incorpora- da dentro del tejido exocrino, está conformada por los islotes de Langerhans, que albergan los distintos tipos de células endocrinas (Slack, 1995).

El páncreas fetal es un órgano activo al final del primer trimestre, momento en el que la placenta es una estructura en evolución hacia la placenta madura; por tanto, los factores histiotróficos que condicionan el desarrollo del embrión son clave para el crecimiento y maduración de la placenta y del páncreas (Kaung, 1994; Reusens y Remacle, 2006). Puede afirmarse que un ambiente anómalo alterará la disponibilidad de sustratos, la angiogénesis y la producción de factores hormonales maternos(Myatt, 2006), que a su vez afectará la producción de factores de transcripción, endocrinos y de variada índole, necesarios para el crecimiento y maduración normal del nuevo ser.

La reducción de la circulación materno-fetal y de la disponibilidad de nutrientes por insuficiencia histiotrófica o placentaria, o por alteraciones en la cantidad y calidad de la dieta, así como por la diabetes materna, alteran no solo el crecimiento del feto, sino, además, la capacidad de las células endocrinas pancreáticas, promoviendo a largo plazo ciertas «incapacidades» que predisponen entre otros aspectos a enfermedades degenerativas como la diabetes mellitus tipo 2 (TD2M) o el síndrome metabólico (Barker, 1995,1999; Serrano-Ríos et al., 2010, 2011).

Actualmente se viene estudiando la histomorfometría del páncreas endocrino durante su desarrollo posnatal en cuyes y alpacas (Rodríguez et al., 2012, 2015), a fin 
de lograr un mayor entendimiento de la fisiología del metabolismo energético en estas especies y de su posible relación con la mortalidad neonatal. Este tema no ha sido trabajado en fetos de alpacas, de allí que el presente estudio tuvo por objetivo determinar la relación que existe entre la glucemia fetal, el desarrollo del páncreas endocrino fetal y la glucemia maternal en alpacas.

\section{Materiales y Métodos}

\section{Animales y Muestras}

Se trabajó con 18 alpacas gestantes, clínicamente sanas de 3-5 años de edad, y sus respectivos fetos, que fueron colectados en el matadero municipal Ninacaca de la Región Pasco, Perú. Los animales fueron agrupados según el tercio de gestación: 3 fetos (F1) y 3 madres (M1) en el primer tercio, 4 fetos (F2) y 4 madres (M2) en el segundo tercio, y 11 fetos (F3) y 11 madres (M3) en el tercer tercio de gestación. La edad fetal fue calculada en base al diámetro biparietal fetal, según la fórmula propuesta por Gazitua et al. (2001).El feto de menor edad fue de 2.3 meses,

Las muestras de sangre fetal y materna se obtuvieron por punción intracardiaca y de la vena yugular, respectivamente. La sangre materna se obtuvo ante-mortem. La sangre fue colectada en tubos de ensayo sin anticoagulante y centrifugada a $3000 \mathrm{~g}$ por $30 \mathrm{~min}$. El suero resultante se almacenó a $-196{ }^{\circ} \mathrm{C}$ en tanque de nitrógeno líquido.

Se recolectaron muestras de páncreas fetal, se diseccionaron en porciones de 1.5$2.0 \mathrm{~cm}^{2}$ y se conservaron en frascos plásticos conteniendo formol tamponado al $15 \%$ $\mathrm{y}$, posteriormente, embebidos en parafina.

\section{Análisis Bioquímico Sanguíneo}

Mediante análisis bioquímico del suero sanguíneo se determinaron los niveles de glucosa en $\mathrm{mg} / \mathrm{dL}$. Se utilizaron kits comercia- les (FAR Diagnostics, Italia) y se siguieron las especificaciones del fabricante. La lectura se hizo en un analizador bioquímico semiautomático (Sinowa, China).

\section{Análisis Histológico}

Las muestras de páncreas en parafina fueron teñidas con Hematoxilina-Eosina (HE). Los islotes de Langerhans (IL) se observaron por microscopía óptica (Carl Zeiss, Alemania), y mediante una cámara digital incorporada AxionCam ERc5s (Carl Zeiss, Alemania) se obtuvieron fotografías a 10X. Se determinó el número de $\mathrm{IL} / \mathrm{mm}^{2}$ de páncreas $\left(\mathrm{N}^{\circ} \mathrm{IL} / \mathrm{mm}^{2}\right)$ y su diámetro $(\mu \mathrm{m})$ con el software de medición ZEN 2012 SP1 (Blue Edition, Carl Zeiss, Alemania).

\section{Análisis de Datos}

Los niveles de glucosa, $\mathrm{N}^{\circ} \mathrm{IL} / \mathrm{mm}^{2}$ y el diámetro de los IL se presentan como promedios y desviaciones estándares. Los datos fueron analizados mediante un análisis de varianza y la determinación de diferencias entre promedios de los tres grupos (tercios) de gestación por la prueba post hoc de Duncan con un nivel de significación del 99.9\%. Asimismo, se determinó la relación entre los valores de glucosa de la madre y los niveles de glucosa fetal, $\mathrm{N}^{\circ} \mathrm{IL} / \mathrm{mm}^{2}$ fetal y el diámetro de los IL fetal mediante el Coeficiente de Correlación de Pearson.

\section{Resultados}

El diámetro de los IL fue de $1.18 \pm 0.01$, $1.36 \pm 0.11$ y $1.56 \pm 0.08 \mu \mathrm{m}$ en fetos durante el primer, segundo y tercer tercio de la gestación, respectivamente ( $<<0.001$; Figura 1$)$. En forma similar, el $\mathrm{N}^{\circ} \mathrm{IL} / \mathrm{mm}^{2}$ fetal fue de 2.36 $\pm 0.04,4.85 \pm 0.58,7.28 \pm 1.38$ en el primer, segundo y tercer tercio de la gestación, respectivamente $(\mathrm{p}<0.001$; Figura 1$)$.

Los niveles de glucosa sanguínea $(\mathrm{mg} / \mathrm{dl})$ en los fetos y las madres fue de 208 \pm 3.5 y $126.5 \pm 2.2$ en el primer tercio de la 

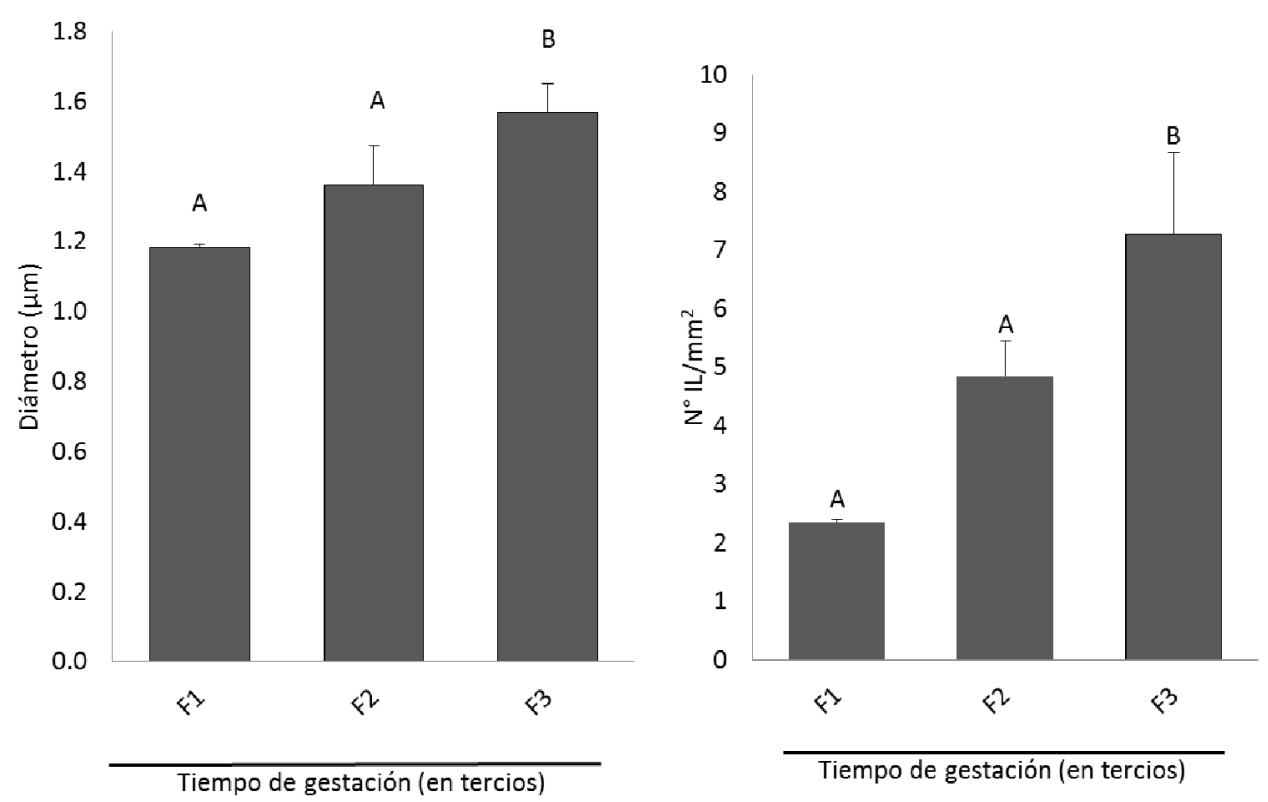

Figura 1. Diámetro de los islotes de Langerhans (IL) y número de islotes de Langerhans por áreas $\left(\mathrm{N}^{\circ} \mathrm{IL} / \mathrm{mm}^{2}\right)$ en fetos de alpacas agrupados en tres tercios de gestación. Letras distintas denotan diferencias significativas evaluadas por ANOVA-Duncan post hoc test $(\mathrm{p}<0.001)$

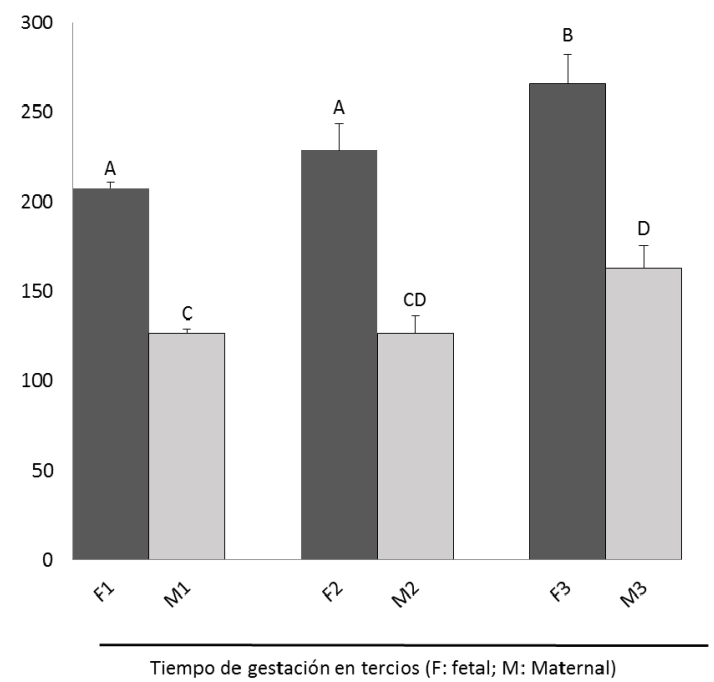

Figura 2. Glucemia fetal y maternal agrupada en tres tercios de gestación en alpacas. Letras distintas denotan diferencias significativas evaluadas por ANOVA-Duncan post hoc test $(\mathrm{p}<0.001)$ gestación, respectivamente; de $229 \pm 14.6$ y $126.5 \pm 9.5$ en el segundo tercio; y de $266 \pm$ 16.2 y $163.2 \pm 12.3$ en el tercer tercio, respectivamente $(\mathrm{p}<0.001$; Figura 2$)$. Las correlaciones entre los niveles de glucosa de las madres con los niveles de glucosa fetal, el $\mathrm{N}^{\circ} \mathrm{IL} / \mathrm{mm}^{2}$ fetal y el diámetro de los IL fetales fueron de $0.98,0.99,0.93$, respectivamente, lo que indicaría una marcada influencia de la glucosa maternal en el desarrollo del páncreas endocrino fetal.

\section{Discusión}

En el presente estudio se pudo determinar la presencia de páncreas endocrino fetal desde el primer tercio de gestación, pudiéndose identificar el número y diámetro de los IL (Figuras 1 y 2). Los valores de estas variables mostraron un incremento significativo 
durante la gestación, lo que indica que el proceso de diferenciación celular en el páncreas endocrino comienza en la etapa fetal. Este proceso se debe a la proteína PDX1, la cual genera la maduración de las células de los IL. Es conocido que PDX-1 se expresa en células precursoras que se encuentran en los compartimentos endocrinos y exocrinos del páncreas y es esencial para su desarrollo (Jonsson et al., 1993; Harrison et al., 1999), para la diferenciación de las células $\beta$ (Ferber et al., 2000; Kojima et al., 2002; Imai et al., 2005) y para el mantenimiento de la madurez y función de las células $\beta$ mediante la regulación de varios genes relacionados con estas células (Petersen et al., 1994; Holland et al., 2005).

Se encontró una alta correlación entre la glucemia maternal y la fetal, la cual aumentó significativamente $(\mathrm{p}<0.001)$ en el último tercio de gestación (Figura 2). La velocidad neta de captación de glucosa en mamíferos está en función directa, tanto de la glucemia materna como del gradiente de concentración de glucosa a través de la placenta (Kulhanek et al., 1974). En el feto de oveja, la utilización de glucosa depende de manera directa de las concentraciones de glucosa materna y fetal, así como de la concentración de insulina fetal (Wilkening y Meschia, 1983). El mecanismo no es a través de la regulación directa del transporte placentario de glucosa sino que es consecuencia de la hipoglucemia fetal que induce por incremento de la captación de glucosa en tejidos fetales, lo que aumenta el gradiente de concentración de glucosa entre la madre y el feto (Kennaugh y Hay, 1987). De esta forma, en el caso de hipoglucemia inducida por el ayuno se observa una caída en la captación umbilical de glucosa y de su utilización, mientras que en casos de hiperglucemia provocada por la infusión de glucosa y elevadas concentraciones de insulina se observa un aumento en la utilización de glucosa en tejidos fetales (Carver et al., 1995).

Los patrones de desarrollo pancreático en nuestro estudio son muy similares a los hallados en otras especies como las ovejas
(Philipp et al., 1978), dado que los patrones de desarrollo fetal son similares y conservados en los mamíferos, desde los roedores hasta los humanos (McMillen y Robinson, 2005; Remacle et al., 2007; Green et al., 2010). Este desarrollo es de vital importancia y continúa durante la etapa posnatal en alpacas y otros mamíferos (Rodríguez et al., 2012, 2015). No obstante, se encontraron algunas diferencias con respecto a la oveja, donde se reportan niveles de glucosa maternal inferiores a los niveles de glucosa fetal en el último tercio de la gestación (Zhang et al., 2011), mientras que en el presente estudio los niveles de glucosa fetal fueron superiores a los maternales en los tres tercios de gestación (Figura 2).

Asimismo, los resultados indicarían que la variación ascendente de la glucemia maternal influye en los crecientes niveles de glucemia fetal y desarrollo pancreático durante la gestación (Figuras 1 y 2). Trabajos en ovejas apoyan dichos hallazgos, pues se ha visto que al incrementar los niveles de glucosa maternal o al disminuirlos por cambios en la dieta, aumentaba o disminuía la glucemia fetal, respectivamente (Wallace $e t$ al., 1997, 2004; George et al., 2010; Redmer et al., 2012).

\section{Conclusiones}

- Los niveles de glucosa fetal-maternal, $\mathrm{N}^{\circ}$ $\mathrm{IL} / \mathrm{mm}^{2}$ y diámetro de los IL son significativamente $(p<0.001)$ mayores en el último tercio de gestación.

- Hay una correlación alta y directa de los valores de glucosa maternal con los valores fetales de glucosa, $\mathrm{N}^{\circ} \mathrm{IL} / \mathrm{mm}^{2}$ y diámetro de los IL, lo que indica su influencia sobre el desarrollo pancreático.

\section{Agradecimientos}

El presente estudio fue financiado con fondos provenientes de la Facultad de Medicina Veterinaria de la Universidad Nacional Mayor de San Marcos y del Proyecto No $173-$ FINCyT-IB-2013, Lima-Perú. 


\section{Literatura Citada}

1. Barker DJ. 1995. Fetal origins of coronary heart disease. BMJ 311:171 -174.

2. Barker DJ. 1999. Early growth and cardiovascular disease. Arch Dis Child 80: 305-307.

3. Carver T, Anderson S, Aldoretta P, Esler A, Hay W. 1995. Glucose suppression of insulin secretion in chronically hyperglycemic fetal sheep. Pediatr Res 38: 754-762.

4. Cebra CK, McKane S, Tornquist S. 2001a. Effects of exogenous insulin on glucose tolerance in alpacas. Am J Vet Res 62: 1544-1547. doi: 10.2460/ajvr. 2001.62.1544

5. Cebra CK, Tornquist SJ, Van Saun RJ, Smith BB. 2001b. Glucose tolerance testing in llamas and alpacas. Am J Vet Res 62: 682-686. doi: 10.2460/ajvr. 2001.62 .682

6. Ferber S, Halkin A, Cohen H, Ber I, Einav Y, Goldberg I, Barshack I, Seijffers $R$, et al. 2000. Pancreatic and duodenal homeobox gene 1 induces expression of insulin genes in liver and ameliorates streptozotocin-induced hyperglycemia. Nat Med 6: 568-572. doi:10.1038/75050

7. Fowler M, Zinkl J. 1989. Reference ranges for hematologic and serum biochemical values in llamas (Lama glama). Am J Vet Res 50: 2049-2053.

8. George LA, Uthlaut AB, Long NM, Zhang L, Ma Y, Smith DT, Nathanielsz PW, Ford SP. 2010. Different levels of overnutrition and weight gain during pregnancy have differential effects on fetal growth and organ development. Reprod Biol Endocrinol 8: 75. doi: 10.1186/1477-7827-8-75

9. Green AS, Rozance PJ, Limesand SW. 2010. Consequences of a compromised intrauterine environment on islet function. J Endocrinol 205: 211-224. doi: 10.1677/ JOE-09-0399

10. Harrison $\mathrm{K}$, Thaler J, Pfaff $S, G u H$, Kehrl J. 1999. Pancreas dorsal lobe agenesis and abnormal islets of
Langerhans in Hlxb9-deficient mice. Nat Genet 23: 71-75. doi: 10.1038/12674

11. Holland A, Hale M, Kagami H, Hammer R, MacDonald R. 2005. Experimental control of pancreatic development and maintenance. Proc Natl Acad Sci USA 99: 12236-12241. doi: 10.1073/pnas.192255099

12. Imai J, Katagiri H, Yamada T, Ishigaki Y, Ogihara T, Uno $K$, Hasegawa Y, et al. 2005. Constitutively active PDX1 induced efficient insulin production in adult murine liver. Biochem Biophys Res Commun 326: 402-409. doi: 10.1016/j.bbrc.2004.11.047

13. Jonsson J, Carlsson L, Edlund T, Edlund H. 1993. Insulin-promoter-factor 1 is required for pancreas development in mice. Nature 37: 606609. doi: $10.1038 / 371606 a 0$

14. Kaung HG 1994. Growth dynamic of pancreatic islet cell populations during fetal and neonatal development of the rat. Dev Dyn 200: 163-175.

15. Kaneko J. 1989. Clinical biochemistry of domestic animals. $4^{\text {th }}$ ed. San Diego: Academic Press. 932 p.

16. Kennaugh JM, Hay WW Jr. 1987. Nutrition of the fetus and newborn. West J Med 147: 435-448.

17. Kim S, MacDonald R. 2002. Signaling and transcriptional control of pancreatic organogenesis. Curr Opin Genet Dev 12: 540-547. doi: 10.1016/S0959-437X(02) 00338-6

18. Kojima H, Nakamura T, Fujita Y, Kishi A, Fujimiya M, Yamada S, Kudo $M$, et al. 2002. Combined expression of pancreatic duodenal homeobox 1 and islet factor 1 induces immature enterocytes to produce insulin. Diabetes 51: 13981408.

19. Kulhanek JF, Meschia G, Makowski EL, Battaglia FC.1974. Changes in DNA content and urea permeability of the sheep placenta. Am J Physiol 226: 1257-1263.

20. Lassen E, Pearson E, Long $P$, Schmotzer W, Kaneps A, Riebold T. 1986. Clinical biochemical values of lla- 
mas: reference values. Am J Vet Res 47:2278-2280.

21. McMillen IC, Robinson JS. 2005. Developmental origins of the metabolic syndrome: prediction, plasticity, and programming. Physiol Rev 85: 571-633. doi: 10.1152/physrev.00053.2003

22. Myatt L. 2006. Placental adaptive responses and fetal programming. $\mathbf{J}$ Physiol 572: 25-30. doi: 10.1113/ jphysiol.2006.104968

23. Noden D, Lahunta A. 1990. Embriología de los animales domésticos. España: Acribia. 422 p.

24. Petersen H, Serup P, Leonard J, Michelsen B, Madsen O. 1994. Transcriptional regulation of the human insulin gene is dependent on the homeodomain protein STF1/IPF1 acting through the CT boxes. Proc Natl Acad Sci USA 91: 10465-10469. doi: 10.1073/ pnas.91.22.10465

25. Philipps A, Carson B, Meschia G, Battaglia $F$. 1978. Insulin secretion in fetal and newborn sheep. Am J Physiol 235: 467-474.

26. Redmer DA, Milne JS, Aitken RP, Johnson ML, Borowicz PP, Reynolds LP, et al. 2012. Decreasing maternal nutrient intake during the final third of pregnancy in previously overnourished adolescent sheep: effects on maternal nutrient partitioning and feto-placental development. Placenta 33: 114-121. doi: 10.1016/j.placenta.2011.11.023

27. Remacle C, Dumortier O, Bol V, Goosse K, Romanus P, Theys N, Bouckenooghe T, Reusens B. 2007. Intrauterine programming of the endocrine pancreas. Diabetes Obes Metab 9 (Suppl 2): 196-209.

28. Reusens B, Remacle C. 2006. Programming of the endocrine pancreas by the early nutritional environment. Int J Biochem Cell Biol 38: 913-922. doi: 10.1016/j.biocel.2005.10.012

29. Rodríguez J, Cueva S, Vásquez M, Lira B, Olivera L, Espinoza J. 2012.
Desarrollo postnatal del páncreas endocrino de cuyes (Cavia porcellus) lactantes. Rev Inv Vet Perú 23: 13-19. doi: 10.15381/rivep.v23i1.877

30. Rodríguez J, Espinoza J, Rojas G Lira $B$, Vásquez M, López-Torres B, Caro C. 2015. Desarrollo de los Islotes de Langerhans en páncreas de crías de alpaca. Rev Inv Vet Perú 26: 381-388. doi: 10.15381/rivep.v26i3.11171

31. Serrano-Ríos M, Gutiérrez Fuentes JA. 2011. Obesity and type 2 diabetes mellitus. The reciprocal impact. En: Obesity. Barcelona: Elsevier. p 215-232.

32. Serrano-Ríos $M$, Reviriego J, Gutiérrez-Fuentes JA. 2010. Classification of diabetes mellitus: criteria for diagnosis. En: Type 2 Diabetes Mellitus. Barcelona: Elsevier. p 1-23.

33. Slack J. 1995. Developmental biology of the pancreas. Development 121: 15691580.

34. Wallace JM, Da Silva P, Aitken RP, Cruickshank MA. 1997. Maternal endocrine status in relation to pregnancy outcome in rapidly growing adolescent sheep. J Endocrinol 155: 359-368.

35. Wallace JM, Milne JS, Aitken RP. 2004. Maternal growth hormone treatment from day 35 to 80 of gestation alters nutrient partitioning in favor of uteroplacental growth in the overnourished adolescent sheep. Biol Reprod 70: 1277-1285.

36. Wilkening RB, Meschia G. 1983. Fetal oxygen uptake, oxygenation, and acidbase balance as a function of uterine blood flow. Am J Physiol 244: H749-H755.

37. Zhang L, Long NM, Hein SM, Ma Y, Nathanielsz PW, Ford S. 2011. Maternal obesity in the ewe results in reduced fetal pancreatic $\beta$-cell numbers in late gestation, and decreased circulating insulin concentration at term. Domest Anim Endocrinol 40: 30-39. doi: 10.1016/j.domaniend.2010.08.004 